\title{
Measuring cycle riding comfort in Southampton using an instrumented bicycle
}

\author{
Shahjahan Miah \\ School of Engineering \\ University of Southampton \\ Southampton, UK \\ 0000-0003-0829-0092 \\ Efstathios Milonidis \\ School of Mathematics, Computer \\ Science and Engineering \\ City, University of London \\ London, UK \\ E.Milonidis@city.ac.uk
}

\author{
Ioannis Kaparias \\ School of Engineering \\ University of Southampton \\ Southampton, UK \\ 0000-0002-8857-1865 \\ Wade Holmes \\ Transport \\ Southampton City Council \\ Southampton, UK \\ Wade.Holmes@southampton.gov.uk
}

\author{
Naumana Ayub \\ School of Mathematics, Computer \\ Science and Engineering \\ City, University of London \\ London, UK \\ Naumana.Ayub.1@city.ac.uk
}

\begin{abstract}
The increased environmental awareness and the rising fuel costs make bicycles a more and more attractive mode of travel for short journeys. Considering the future prospect of this mode of transportation and the great advantages that it offers in terms of space consumption, health and environmental sustainability, several city authorities worldwide are presently undertaking schemes aiming at improving cycling infrastructure. The aim of the present study is to monitor the impact of such schemes on the riding comfort of cyclists, as expressed by the, usually lower, quantity and magnitude of vibrations occurring as a result of cycling over pavement defects. Millbrook Road East in the western edge of the city center of Southampton is used as a case study, where vibration measurements are taken by means of an instrumented bicycle during periods before and after a redevelopment scheme involving the resurfacing of the road pavement. The results show a clear overall improvement in cycling comfort postredevelopment, with statistically significant reductions in both the number of high severity vibrations and of their magnitude in "typical" cycling trips taken on the road. However, instances of finishing "snags" in some parts of the surface appear to introduce new minor defects (e.g. around manholes) that are not visible to the naked eye, and these still have some negative effect on the riding experience. Moreover, the study highlights the detrimental impact that widespread pavement defects can have on riding comfort, which affect cyclists of all ages, abilities and styles.
\end{abstract}

Keywords-severity, instrumented bicycle, riding comfort, accelerometer, survey data, cyclists' perceptions and traffic calming schemes.

\section{INTRODUCTION}

Cycling is an increasingly popular mode of travel in cities due to the great advantages that it offers in terms of space consumption, health and environmental sustainability. Yet, the low perceived safety and riding comfort of cyclists is a hurdle to the desired uptake of cycling as an alternative to the car, with a particular deterrent being poorly maintained infrastructure, including road pavement defects, such as fretting, raveling, rutting and potholes. As a result, several city authorities worldwide are presently undertaking schemes aiming at improving cycling infrastructure.

One such scheme has recently been implemented in Millbrook Road East (MRE) in the UK port city of Southampton. Opinion surveys with cyclists regularly using MRE had identified that a series of pavement defects on the road surface affected the overall cycling experience, which was generally rated very poorly. This prompted the local authority to resurface the road, with works having been recently completed. However, even though the perception of the cyclists continues to be analyzed through surveys with cyclists post-completion of the works, establishing its relation with actual objective riding comfort measurements is challenging.

The aim of this study is, hence, to investigate changes in riding comfort as a result of the resurfacing of the MRE site on the basis of objective vibration measurements collected using the iBike instrumented bicycle [1]. This is an advanced ITS research tool, equipped with an absolute encoder and offthe-shelf micro-electromechanical systems (MEMS) sensors (Hall Effect sensor, gyroscopes, accelerometers, magnetometers, temperature and pressure sensors), as well as with a GoPro camera. The study involves riding the iBike on MRE at periods before and after resurfacing to collect mapped vibration data from the sensors, and then drawing comparisons between the two periods with respect to the number and magnitude of "hits", both in terms of individual locations/hot-spots and the road in its entirety.

The rest of the paper is structured as follows: Section II reviews relevant literature on measuring riding comfort using various parameters, methods and tools. Section III then describes methodology of the study, which includes the instrumentation and sensor configuration of the bicycle, the study site and the data collection and processing methods employed. The results of the study and their interpretation are presented in Section IV. Finally, Section V concludes the paper and identifies limitations and areas of further work.

\section{BACKGROUND}

Much previous research has confirmed that the vibrations experienced by a cyclist can have an adverse effect on the overall cycling comfort, and has therefore attempted to analyze it. For instance, Bíl et al. [2] proposed a simple approach to capture vibration data using a GPS sensor and accelerometer in order to analyze the discomfort caused by vibrations. GPS was used to gather information on the cyclist's positions and the bicycle vibrations were recorded using the accelerometer. The Dynamic Comfort Index (DCI) was proposed to classify cycling comfort, which was inversely proportional to the power of acceleration; the higher the value of the DCI, the more comfortable the cycle track was. Bíl et al. computed DCI values at second-long intervals, which, combined with the GPS position, enabled the mapping of comfort measurements with fairly good localization accuracy. 
On the other hand, Calvey et al. [3] implemented an instrumented bicycle, along with a specially designed questionnaire, to develop an understanding of the condition of cycle paths in Edinburgh. Cyclist perceptions obtained through the questionnaire were combined with the data collected from the instrumented bike (mainly accelerometer data) to determine the rating for a particular path. It was aspired that this information would be useful to decisionmakers when it came to effectively use the limited resources for maintenance, repair and renewal of cycle tracks. Similarly, Olieman et al. [4] measured the level of vibrations experienced by a cyclist using acceleration sensors, which were mounted, at four different places on a bicycle. The results showed that the road surface, the speed of the bicycle and the pressure in the tires significantly affected the level of vibrations induced to the frame and fork of the bicycle, thus directly impacting comfort.

Zang et al. [5] used the GPS and accelerometer sensors of a smartphone, which was mounted on a bicycle, to measure the roughness of pedestrian and bicycle lanes. They proposed the so-called "International Roughness Index" (IRI) to describe their results, which was calculated using the total distance travelled by the cyclist and the vertical acceleration. The travel distance was computed by measuring the speed of the bicycle at each sampling interval, which was directly obtained from the GPS sensors, whereas the vertical acceleration was determined from the tri-axial acceleration values collected by the accelerometer in each sampling interval. They further proposed an algorithm, which took the measured vertical acceleration, a certain acceleration threshold and a time gap threshold, as inputs to detect potholes and humps, and to identify their locations.

Gao et al. [6] analyzed the correlation between the cycle's vibrations and the cyclist's perception of comfort. The perception was established by means of a questionnaire, while the vibrations were calculated via a "Dynamic Cycling Comfort" (DCC) measuring system, which relied on an accelerometer, a GPS logger and a smartphone to record vibrations. Correspondingly, Ayachi et al. [7] carried out an online survey to find out which factors influence cyclist comfort. Their results showed that the components of the bicycle, the road surface, the weather conditions, the cyclist's position, and any adjustments and body parts, are all relevant in determining cycling comfort.

It can be, hence, concluded, that different parameters and methods have been used in the past to measure the riding comfort. Nevertheless, they have all been limited in terms of two main aspects:

1. Several studies did not attempt to localize the pavement defects that caused the cycling discomfort, and the ones that did used exclusively GPS. However, GPS can only achieve a positioning accuracy of at best $10-20 \mathrm{~m}$ in urban areas due to the presence of features such as urban canyons, and while this may be enough to approximately pinpoint defects within the scale of an entire network, it is not sufficient when it comes to more accurately localizing them at the individual street level. This may be crucial for defects that may not be visible to the naked eye.

2. Some of the studies attempted to explicitly bring in cyclist perceptions in the measurement of cycling comfort, and this was done mainly through questionnaires. However, while such approach may enable obtaining a fairly good overall picture of the anticipated or experienced riding comfort before or after a ride, it does not allow the accurate linkage of specific defects with perceived occurrences of discomfort, as the latter cannot be captured in real-time (i.e. at the time when they actually happen).

The present study attempts to address these two limitations by using the iBike instrumented bicycle, which enables accurate cyclist localization $(50 \mathrm{~cm})$ by means of MEMS sensors [8], on the MRE case study in Southampton. Vertical acceleration data, as obtained through the iBike's onboard accelerometers, are transformed to hit counts and magnitude values, are categorized into three severity levels, and are accurately mapped on the road surface. In addition, the severity based on the sensor data is then compared with real-time cyclist comfort perception data, which are also recorded using the iBike while it is being ridden.

\section{METHODOLOGY}

This section introduces the iBike and describes its instrumentation and sensor configuration. It then goes on to introduce the MRE study site, which is used as a case study, and to describe the data collection and processing tasks that are employed to obtain cyclist comfort information from the raw sensor data.

\section{A. The iBike instrumented bicycle}

The iBike is an instrumented bicycle capable of monitoring several key variables related to cycling, such as speed, steering and acceleration, through an on-board bespoke measuring and logging system. Although the iBike was originally developed to enhance the positioning accuracy of cyclists (related studies can be found in [8]), the measurement from the iBike can also be employed to monitor the road pavement surface quality. For instance, the acceleration data can be related to surface quality and can be mapped with the positioning data from the iBike. Fig. 1 illustrates the actual instrumented bicycle with its sensor configuration.

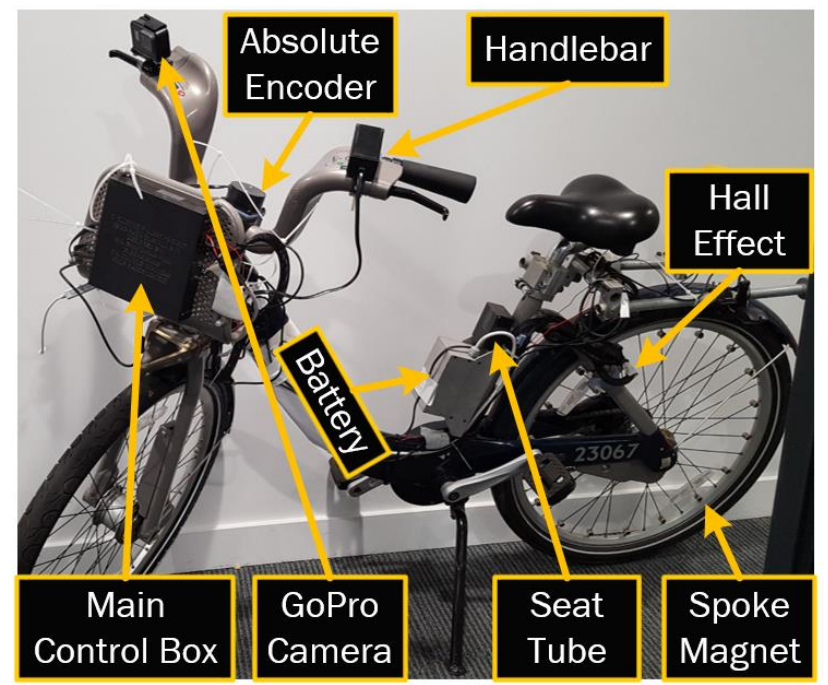

Fig. 1. The instrumented bicycle (iBike)

The housing of the handlebar sensors includes a GY80 Inertial Measurement Unit (IMU), which incorporates a three-axis accelerometer, three-axis gyroscope, three-axis magnetometer, temperature and pressure sensors. The 
housing also includes a light-emitting diode (LED) and a push button. The LED is used to indicate the status of measurement and logging system and the push button is used to start and stop the data acquisition, as well as to place a realtime flag (marker) on the data. This function of the iBike is employed to record the rider's perceptions in this study. For example, the cyclist can press the push button while the bicycle is being ridden to indicate the location of a pothole or a surface defect, and the iBike system will record this data along with the measurements from the sensors with a timestamp in real-time. Thus, the data can be used as a measure of a cyclist's comfort perception.

The seat tube enclosure houses another set of accelerometer and gyroscope configuration, and these are used to monitor the rear frame acceleration and angular rate respectively. The Hall Effect and the absolute encoder sensors are used to measure the revolutions of the rear wheel and the steering angle respectively, while the main control box incorporates an Arduino Mega 2560 board along with various electronic components and micro SD memory card. In addition, a GoPro camera is installed on the bicycle for the purpose of visual validation of the journeys. The iBike's full system architecture is shown in Fig. 2.

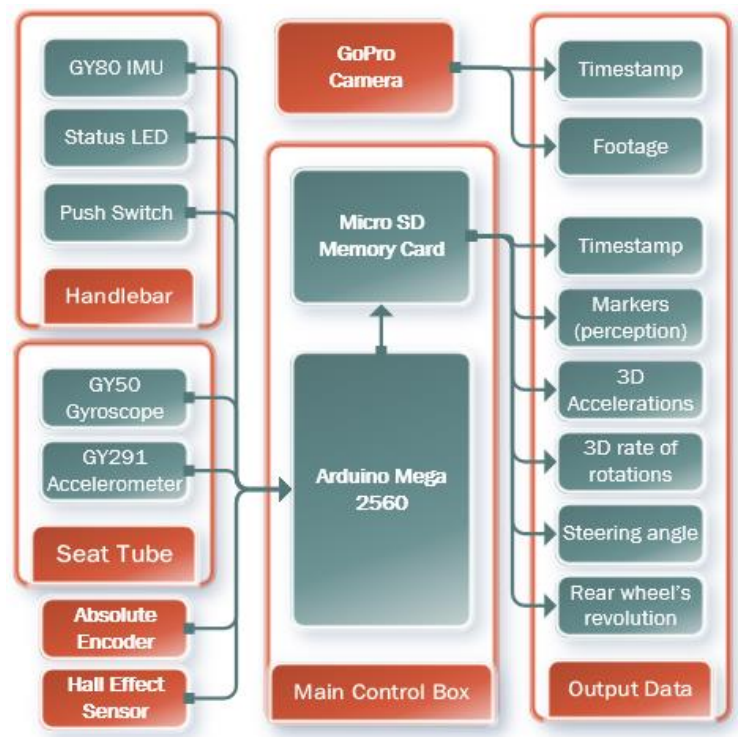

Fig. 2. iBike measurement system architecture and output data

In order to establish riding comfort and demonstrate the iBike's capability in this respect, only the timestamp, markers and vertical acceleration data are analyzed in this study. The authors believe that cycle riding comfort is very likely also influenced by the rider's behavior, and this could include a variation of speeds and steering angles in order to avoid a pothole or surface defect. However, this is beyond the scope of the present study and the authors envisage analyzing these other parameters in a subsequent publication.

\section{B. Study site}

As part of Southampton City Council's strategic goals, portrayed in the recently published 10-year cycling plan [9] and the successful "My Journey" behavior change program [10] in Southampton, it is envisaged for cycling to play a prominent role in the city, and as such, a number of ambitious targets are foreseen. These include the doubling of the cycling mode share by 2020 , as well as the building of cycling infrastructure along three main corridors radiating East, North and West of the city center (Fig 3).

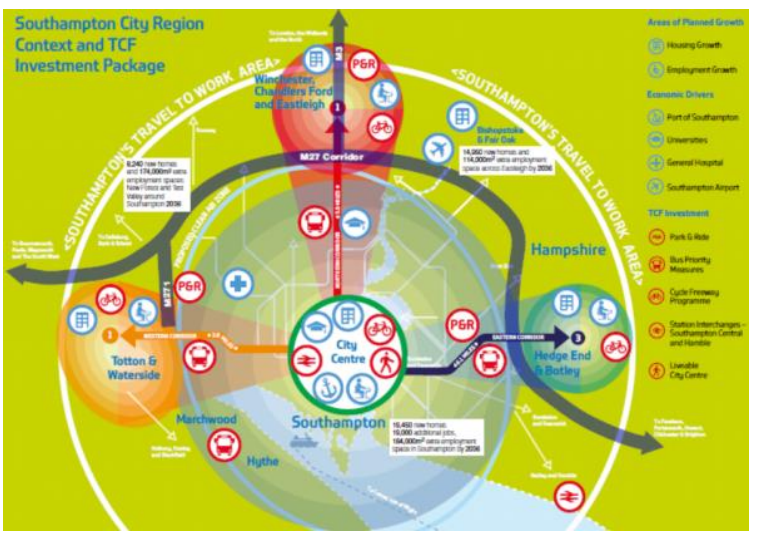

Fig. 3. Investment in cycling infrastructure in Southampton (Source: Southampton City Council)

Forming part of the Western corridor, MRE is a $1 \mathrm{~km}$ long road stretch located on the western edge of the city center of Southampton. In a number of recent opinion surveys carried out by Southampton City Council in order to gather insight on the cyclists' perception of MRE, the majority of the respondents rated the cycling condition of MRE as "poor" and expressed dissatisfaction with it. The main reasons identified for this perception included: potholes; poor maintenance; vehicles parked along the road; vehicle speeds; and the number of vehicles using the road.

As a result of the feedback, Southampton City Council decided to redevelop the MRE site to a "Low Traffic Neighborhood Scheme". In essence, the redevelopment scheme was to proceed in two phases, with the first phase concentrating on resurfacing the road to eliminate potholes and other surface defects, and the second one entailing the introduction of a wide range of traffic management measures to restrict vehicle traffic and facilitate cyclists (and by extension also pedestrians). These include: introduction of a modal filter, which would only allow pedestrians and bicycles to pass through; implementation of a short one-way restriction; provision of enhanced landscaping and seating; changing of junction priority rules to give cyclists along MRE right of way; and widening of the cycle paths (Fig. 4).

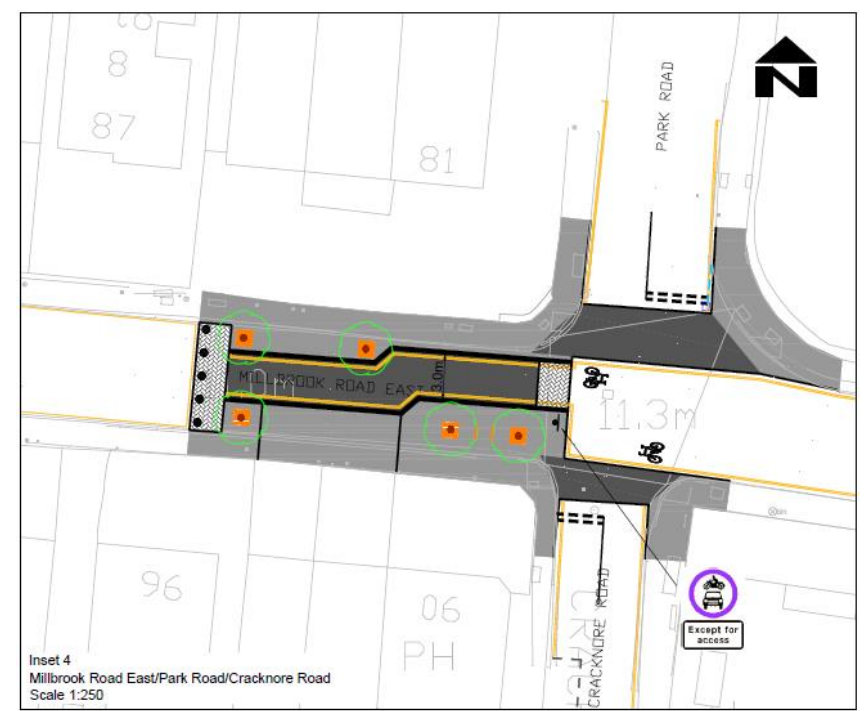

Fig. 4. Modal filter and short one-way conversion along MRE (Source: Southampton City Council) 
The present study focuses on the first phase of the MRE redevelopment scheme, i.e. the resurfacing. The data collection involves a single cyclist riding the iBike on MRE, first before the start of the resurfacing works, and then after their completion. This results in two sets of mapped sensor, data corresponding to a total of 21 runs for each of the beforeand after-case. Each run is considered either from start to end or vice-versa, such that a completed round trip along MRE, highlighted in pink in Fig. 5, is considered as two runs.

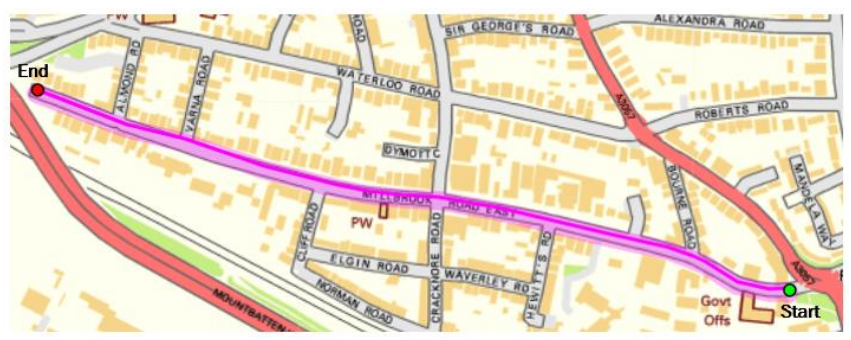

Fig. 5. Survey route: MRE, Southampton, UK

\section{Data collection and processing}

Cycling comfort is dependent on several factors, which range from the bicycle's component parts (i.e. handlebar, frame, saddle etc.), to environmental factors (i.e. weather, type of road, slope, etc.) and to factors related to the cyclist themselves (i.e. sitting position, adjustments, body parts, etc.) [7]. The roughness of the road pavement surface, however, as experienced by the cyclist is one of the most important factors influencing the overall riding comfort along a road [2], and usually plays an important part in cyclist route choices.

In this study, the longitudinal roughness of the surface is measured using the vertical acceleration data from the iBike's accelerometer. Several steps are required to convert the raw measurement data into acceleration values, and these are demonstrated in Fig. 6. As can be seen, the raw data from the accelerometer is first converted into a vertical g-force, and since the data already includes the normal gravitational force $\left(1 g=9.81 \mathrm{~ms}^{-2}\right)$, it needs to be removed here by reducing the g-force by $1 g$.

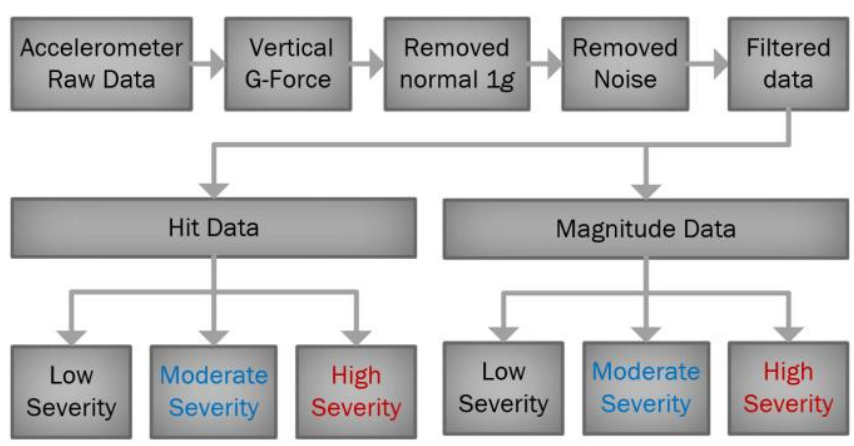

Fig. 6. Extraction of vibration values from the accelerometer's raw data

Furthermore, accelerometers are susceptible to noise and in this case, some of the noise is associated with the riding and mechanical noise from the bike, such as noise from pedaling. In order to remove this noise, an experiment has been conducted on a smooth surface and it has been found that approximately $0.2 g$ is generated under the normal conditions of riding the iBike even on a smooth surface, i.e. regardless of the surface roughness. This can also be observed from Fig. 7, where all the hits below the black line are considered to be noise.
Data from the three-axis accelerometer located inside the seat tube enclosure are extracted using the iBike system and then processed using the procedure outlined in order to compute the number of hits within a threshold and their magnitude values. Fig. 7 presents the vertical acceleration data from a single run conducted at the survey location and after taking the absolute value and removing the normal $1 \mathrm{~g}$ from them. Each pink solid dot represents a vibration hit and it is also associated with a magnitude value, which is used to categorize it into either "low", "moderate" or "high" severity. In the present study, the threshold ranges shown in Table I are adopted based on a fine-tuning exercise carried out by analyzing the GoPro camera's footage, the data from the accelerometer and the markers denoting cyclist perceptions. A more elaborate study seeking to establish the threshold values for these categories in a systematic way is currently under way; however, this is beyond the scope of this paper.

TABLE I. SEVERITY THRESHOLDS AND CATEGORIES

\begin{tabular}{|c|c|c|}
\hline Low Severity $[\boldsymbol{g}]$ & Moderate Severity $[\boldsymbol{g}]$ & High Severity $[\boldsymbol{g}]$ \\
\hline values from 0 to 0.4 & values from 0.4 to 0.8 & values greater than 0.8 \\
\hline
\end{tabular}

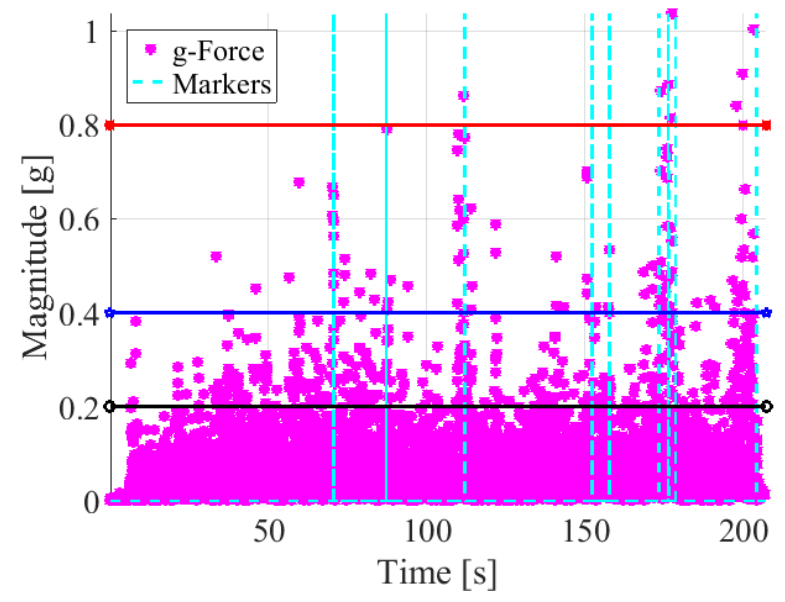

Fig. 7. Pre-resurfacing accelerometer data with markers and thresholds

\section{RESULTS}

This section reports on the analysis of the cyclist comfort data collected from MRE using the iBike system, as outlined in Section III. Comparisons between the before- and afterresurfacing cases are made in terms of both vibration hit counts and their magnitude values. Finally, the effect of speed on riding comfort is also discussed using the survey data.

\section{A. Overall cycling comfort}

Fig. 8 and Fig. 9 show "heatmaps" of the real-time perceived cycling comfort before and after resurfacing of the MRE for all runs carried out. The pink dots represent the longitude and latitude coordinate data established using the iBike localization system (i.e. the trajectory), whereas the cyan blue crosses denote occurrences of uncomfortable vibration, as identified by the rider in real-time. As can be observed from the mapped data, instances of perceived uncomfortable vibrations have greatly reduced postresurfacing, so cycling comfort has overall improved considerably. Nevertheless, Fig. 9 also shows that there are still certain locations along the road where cycling comfort has not changed even after resurfacing. This could be due to the fact that certain parts of the road have either not been resurfaced or modified, possibly in preparation for the 
improvement works of the second phase of the project traffic calming measures.

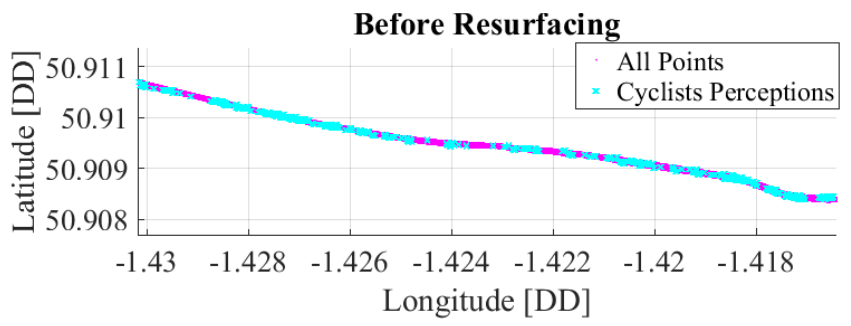

Fig. 8. Pre-resurfacing: perceived cyclist comfort along MRE

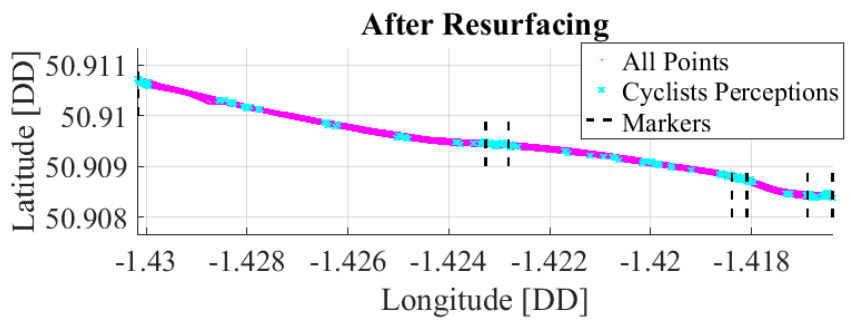

Fig. 9. Post resurfacing: perceived cyclist comfort along MRE

Subsequently, the vibration hit data with their magnitude and position values found through the analysis of the accelerometer data for all runs of the before- and afterresurfacing cases are depicted in Fig. 10, grouped by severity category. The locations of Markers $\mathrm{A}$ and $\mathrm{C}$ in the figure have been identified as parts of the road that have not been resurfaced, whereas the location of Marker B represents a speed hump. It is apparent that the number of high severity hits has considerably decreased in the after case, and visual examination of the data shows that it matches cyclist's perception depicted in Fig. 8 and Fig. 9.
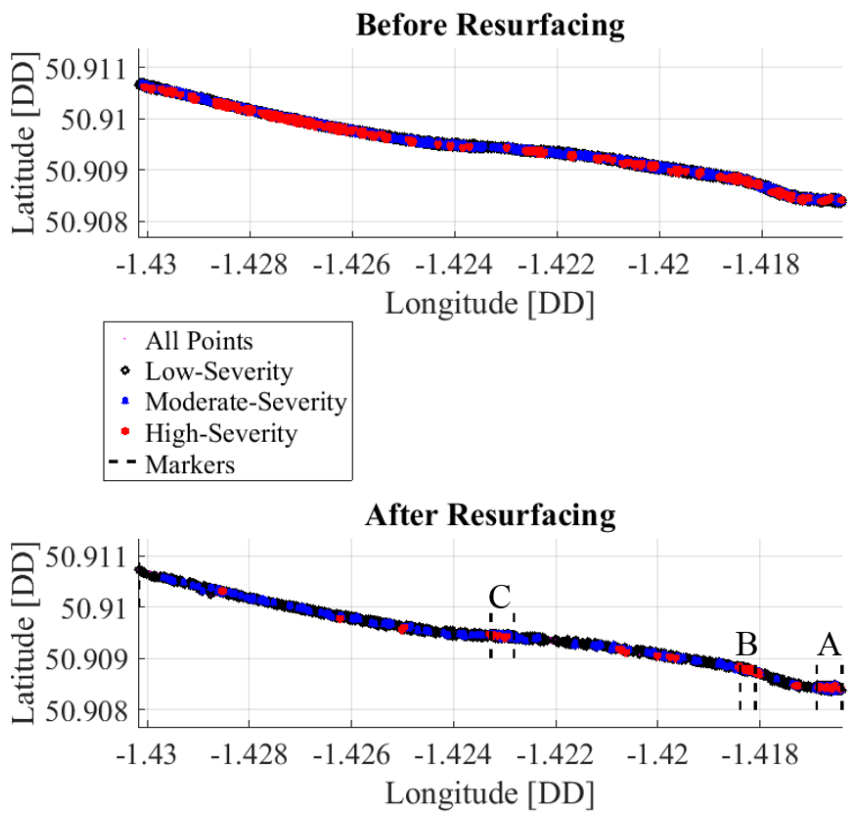

Fig. 10. Hit severity pre- and post-resurfacing along MRE

\section{B. Visual examination of defects}

Examples of road pavement defects on MRE causing low, moderate and high severity hits before and after resurfacing are given in Table II. In the before case, some of the main discomfort instances were linked to pavement edge cracking $(a$ and $d)$, potholes of low, moderate and high severities $(b$ and $c$ ), manholes $(e)$ and longitudinal joint and alligator

cracking of high severity $(f)$. In the after case, most of these defects have been repaired through the resurfacing of MRE. However, instances of finishing "snags" in some parts of the surface appear to introduce new minor defects $(g, h$ and $i)$ that are not visible to the naked eye, and these still have some negative effect on the riding experience, as demonstrated both by the rider themselves and the analysis of the sensor data presented in Fig. 9 and Fig. 10 (after case).

TABLE II. IMAGES OF DEFECTS ALONG MRE PRE AND POST RESURFACING

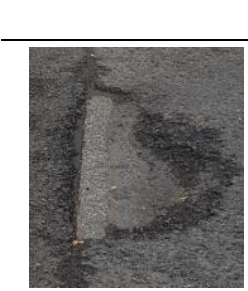

(a)

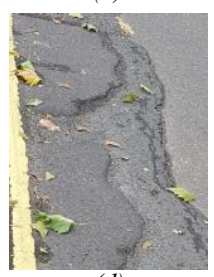

(d)

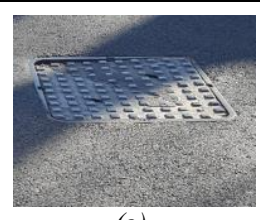

(g)

\section{Before Resurfacing:}

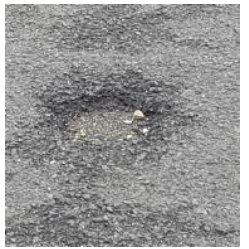

(b)

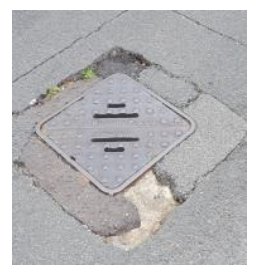

(e)

After Resurfacing:

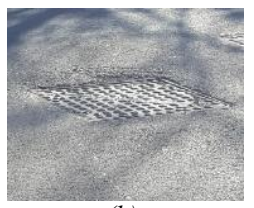

(h)

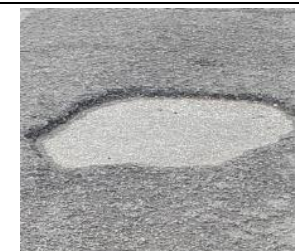

(c)

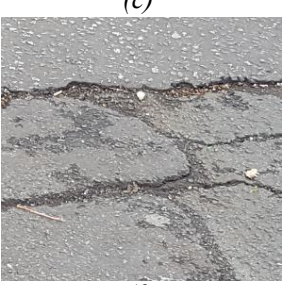

(f)

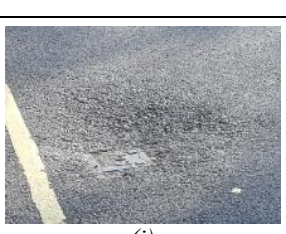

(i)

\section{Vibration hit counts}

Fig. 11 shows the average number of hits for each severity category on MRE before and after resurfacing. Confirming the results of the previous sub-sections, it can be clearly seen that high-severity hits have on average reduced to single digits per run, while medium- and low-severity hit occurrences have reduced to a fifth and a quarter of their preresurfacing values respectively.

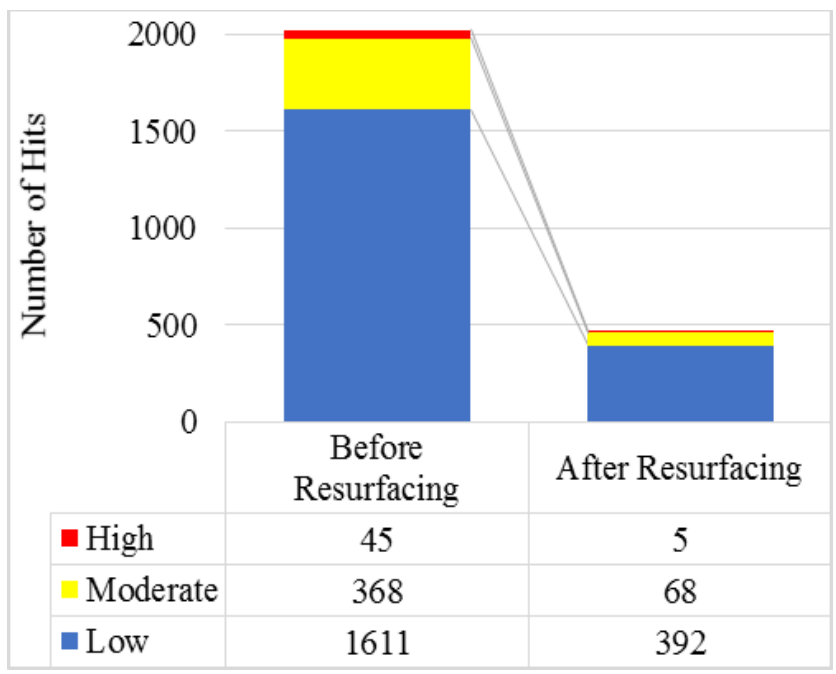

Fig. 11. Average number of vibration hits for pre-and-post-resurfacing

The full data of the number of hits for each severity category and the journey time for each run are then presented 
in Table III. As can be seen, the resurfacing of MRE has significantly reduced the number of vibration hits across the three severity levels for all 21 runs in the after case.

Furthermore, it is also clear from Fig. 12 that on average the journey time for the after-case has reduced by 9 seconds along the $1 \mathrm{~km}$ surveyed route. This suggests that in the aftercase the cyclist has been able to ride faster along the route because of the reduction of the discomfort caused by surface defects.

TABLE III. COMPARISON OF SEVERITY AND JOURNEY TIME PRE- AND POSTRESURFACING OF MRE

\begin{tabular}{|c|c|c|c|c|c|c|c|c|}
\hline \multirow{2}{*}{ Index } & \multicolumn{4}{|c|}{ Before Resurfacing } & \multicolumn{4}{|c|}{ After Resurfacing } \\
\hline & Low & Mod. & High & Time [s] & Low & Mod. & High & Time [s] \\
\hline 1 & 1597 & 336 & 33 & 356 & 226 & 49 & 3 & 273 \\
\hline 2 & 2024 & 391 & 50 & 327 & 167 & 63 & 5 & 292 \\
\hline 3 & 1184 & 236 & 26 & 279 & 127 & 19 & 0 & 298 \\
\hline 4 & 1628 & 342 & 21 & 261 & 141 & 10 & 2 & 311 \\
\hline 5 & 1338 & 383 & 42 & 278 & 126 & 19 & 0 & 286 \\
\hline 6 & 1622 & 346 & 39 & 294 & 115 & 30 & 0 & 301 \\
\hline 7 & 1136 & 169 & 8 & 283 & 214 & 17 & 4 & 268 \\
\hline 8 & 1478 & 284 & 32 & 283 & 158 & 36 & 2 & 299 \\
\hline 9 & 1300 & 350 & 40 & 295 & 107 & 20 & 0 & 309 \\
\hline 10 & 1626 & 427 & 55 & 290 & 464 & 61 & 10 & 258 \\
\hline 11 & 1621 & 387 & 64 & 331 & 704 & 176 & 12 & 207 \\
\hline 12 & 2006 & 476 & 41 & 351 & 514 & 114 & 5 & 231 \\
\hline 13 & 1598 & 282 & 52 & 344 & 711 & 124 & 1 & 221 \\
\hline 14 & 1953 & 403 & 53 & 350 & 530 & 118 & 12 & 237 \\
\hline 15 & 1818 & 457 & 51 & 310 & 688 & 101 & 9 & 218 \\
\hline 16 & 2025 & 519 & 78 & 302 & 449 & 74 & 3 & 236 \\
\hline 17 & 1803 & 462 & 74 & 296 & 402 & 60 & 6 & 253 \\
\hline 18 & 1617 & 301 & 38 & 348 & 727 & 104 & 6 & 224 \\
\hline 19 & 1215 & 286 & 29 & 233 & 549 & 86 & 8 & 244 \\
\hline 20 & 1414 & 380 & 49 & 255 & 478 & 52 & 3 & 248 \\
\hline 21 & 1826 & 510 & 68 & 274 & 643 & 88 & 9 & 233 \\
\hline Average & 1611 & 368 & 45 & 302 & 392 & 68 & 5 & 259 \\
\hline
\end{tabular}

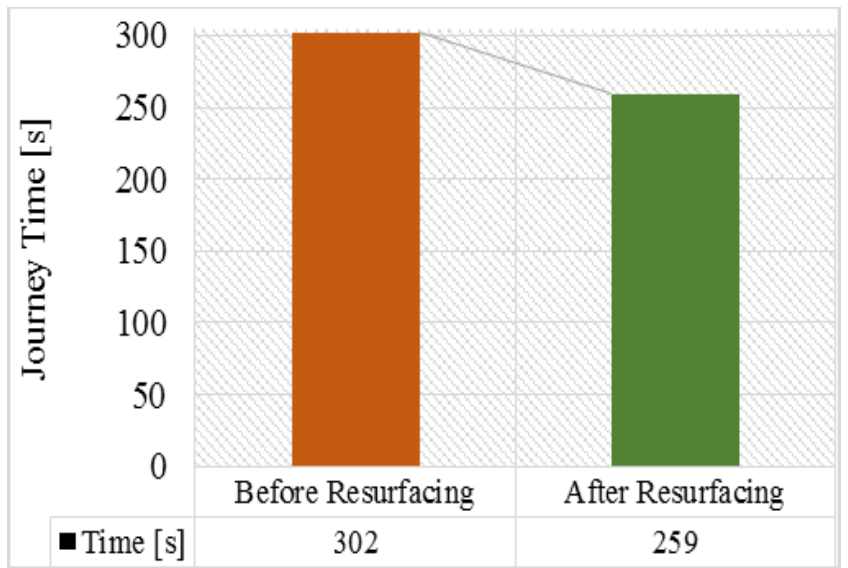

Fig. 12. Average journey time along MRE pre-and-post-resurfacing

\section{Vibration hit magnitude}

The average, standard deviation, maximum and minimum of the magnitude of each vibration hit severity category and as a whole are computed for each run separately, and the mean values of all these are presented in Table IV. The results suggest that on average the magnitude has decreased by $0.023 \mathrm{~g}$ across all hit severity levels, and this finding is statistically significant to the 0.05 level $(p=0.000)$. This represents a $7 \%$ reduction, and it can be clearly observed when plotting the cumulative distribution function (CDF) of the magnitude in Fig. 13, as the curve corresponding to the post-resurfacing case is shifted to the left compared to the pre-resurfacing one.

TABLE IV. PRE AND POST RESURFACING MEANS OF AVERAGE, STANDARD DEVIATION, MAXIMUM AND MINIMUM MAGNITUDE VALUES FOR ALL 21 RUNS

\begin{tabular}{|c|c|c|c|c|c|c|c|c|}
\hline & \multicolumn{4}{|c|}{ Before (average) } & \multicolumn{4}{|c|}{ After ((average) } \\
\hline & $\begin{array}{l}A v . \\
{[g]}\end{array}$ & $\begin{array}{c}\text { S. Dev. } \\
{[g]}\end{array}$ & $\begin{array}{c}\operatorname{Max} . \\
{[g]}\end{array}$ & $\underset{[g]}{M i n .}$ & $\begin{array}{l}A v . \\
{[g]}\end{array}$ & $\begin{array}{c}\text { S. Dev. } \\
{[g]}\end{array}$ & $\underset{[g]}{\operatorname{Max} .}$ & $\underset{[g]}{\text { Min. }}$ \\
\hline Low & 0.268 & 0.053 & 0.399 & 0.200 & 0.263 & 0.051 & 0.395 & 0.201 \\
\hline Mod. & 0.520 & 0.099 & 0.789 & 0.401 & 0.524 & 0.098 & 0.761 & 0.408 \\
\hline High & 1.052 & 0.272 & 1.834 & 0.809 & 1.003 & 0.132 & 1.155 & 0.862 \\
\hline All & 0.331 & 0.162 & 1.834 & 0.200 & 0.308 & 0.129 & 1.066 & 0.201 \\
\hline
\end{tabular}

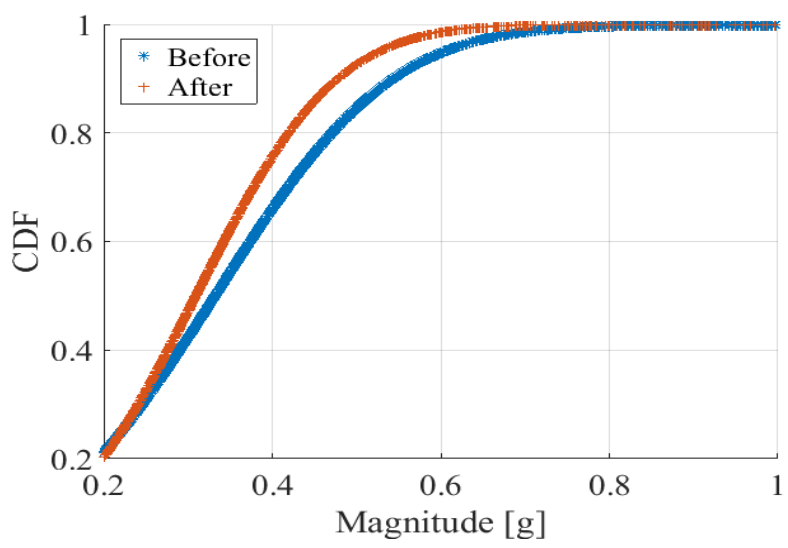

Fig. 13. Cumulative distribution function of all hits before and after

Looking at the averages of the magnitude of the hits of the different severity categories in Table IV, it can be observed that the overall reduction is largely due to a $0.005 \mathrm{~g}$ drop in the magnitude of the low-severity hits, which is also statistically significant to the 0.05 level $(p=0.000)$. The average magnitude of the high-severity hits also appears to have decreased, whereas that of moderate-severity hits seems to have slightly increased; however, these findings are not statistically significant to the 0.05 level ( $p=0.112$ and $p=$ 0.245 respectively).

The analysis of the average maximum and average standard deviation of the magnitude also delivers some interesting conclusions. In the case of the maximum values, as can be observed in Fig. 14, there appears to be a reduction in the average maximum hit magnitude per run, and this can be attributed to a clear reduction in the maximum magnitude of the high-severity hits. A similar pattern is also observed in the case of the average standard deviation (Fig. 15), where it appears that a slight decrease in overall hit magnitude variability is attributed to a clear reduction in the variability of the high-severity hits.

These confirm the finding that the resurfacing has overall improved cycling comfort on MRE. This is achieved by not only reducing the total number of vibration hits per journey, but also by "softening" the magnitude of these hits. More specifically, it has predominantly reduced the magnitude of the many low-severity hits, but has also "taken off the edge" of the fewer high-severity hits. 


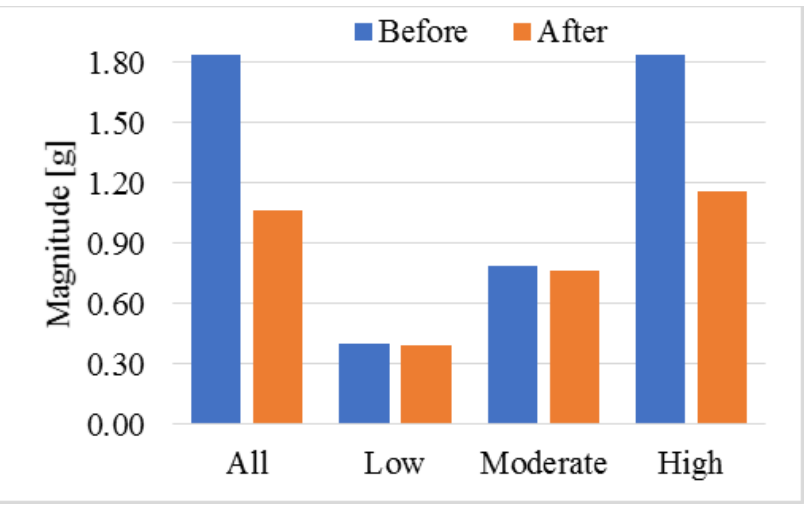

Fig. 14. Average maximum magnitude pre-and-post-resurfacing

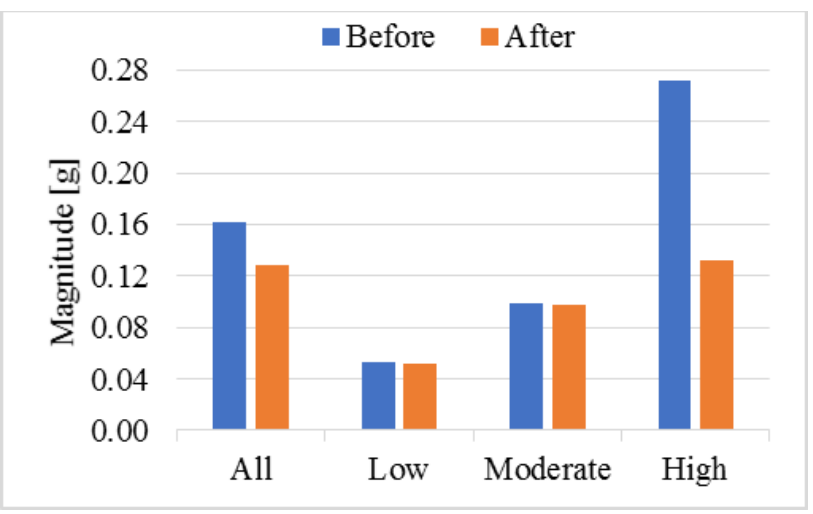

Fig. 15. Average standard deviation of magnitude pre-and-post-resurfacing

\section{E. Vibration and riding speed}

It has been identified in the literature that riding speed is one of the most important factors affecting comfort, as higher riding speed generally impedes the cyclist's ability to avoid pavement defects, and therefore results in more vibration. It is, therefore, interesting to explore whether the pavement condition may impact the correlation of these two parameters.

Fig. 16 presents the relation between cycling speed and number of hits before resurfacing for the three-hit severity categories; each data point on the three graphs represents one experiment run on MRE. As can be observed, there appear to be more low-severity hits when the cycling speed is low, but as the speed increases, the number of low-severity hits reduces; this is an unexpected finding. A possible explanation could lie in the fact that, previously low-severity, hits may be becoming moderate- or even high-severity ones, because many of the existing widespread potholes, humps and other defects are hit at higher speed. A similar, yet considerably weaker, association appears to exist between the number of moderate- and high-severity hits and the cycling speed. This suggests that the widespread and largely unavoidable pavement defects on the pre-resurfacing MRE pavement result in the number of moderate- and high-severity hits being unaffected by the cycling speed.

Considering the post-resurfacing case of MRE, the relation between speed and number of hits is depicted in Fig. 17. As can be observed, there appears to be an opposite relation between the two parameters compared to the beforecase, as higher travel speed is associated with a higher number of hits across all three severity categories. This can be attributed to the fact that most pre-resurfacing defects on MRE have been repaired/removed, and the selected few ones remaining are avoidable by swerving. As such, when riding slowly, it is possible to swerve around the defects, which results in lower numbers of hits, but when riding faster, it is more difficult to avoid the defects, so a higher number of hits occurs. Thus, post-resurfacing cycling comfort appears to be more strongly dependent on cycling speed than in the beforecase, where speed plays a less important part.

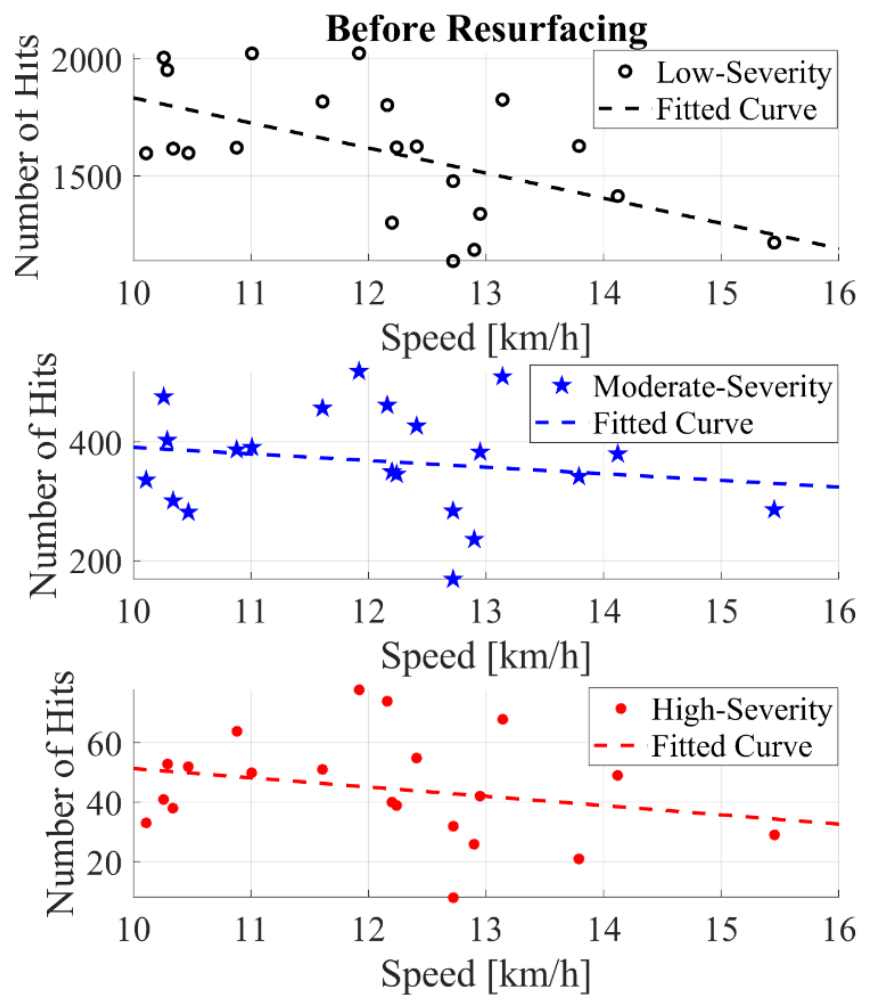

Fig. 16. Pre-resurfacing: cycling speed and number of hits
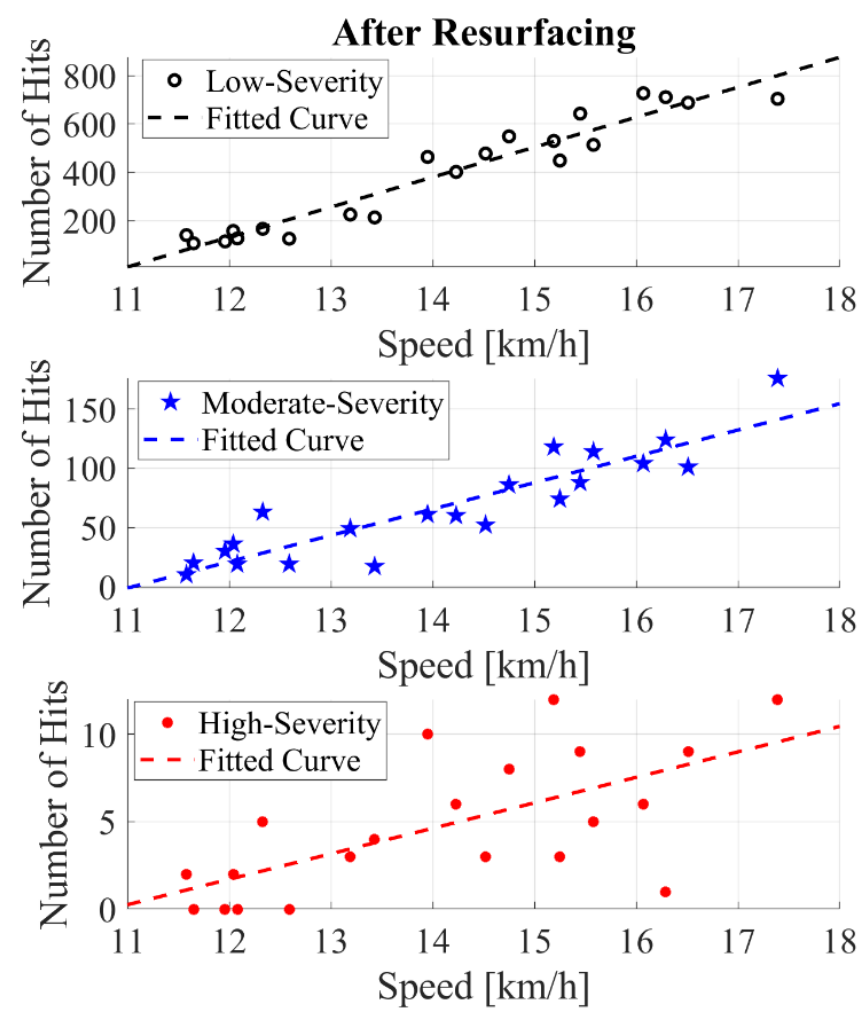

Fig. 17. Post-resurfacing: cycling speed and number of hits 
TABLE V. VIBRATION HITS AND SPEED CORRELATION

\begin{tabular}{|l|c|c|c|c|}
\hline & $\begin{array}{c}\text { Low } \\
\text { Severity }\end{array}$ & $\begin{array}{c}\text { Mod. } \\
\text { Severity }\end{array}$ & $\begin{array}{c}\text { High } \\
\text { Severity }\end{array}$ & $\begin{array}{c}\text { All } \\
\text { Severity }\end{array}$ \\
\hline Pre-resurfacing & -0.55 & -0.18 & -0.25 & -0.48 \\
\hline Post-resurfacing & 0.96 & 0.91 & 0.66 & 0.97 \\
\hline
\end{tabular}

The correlation coefficients for the before- and afterresurfacing hits and speed in Table $\mathrm{V}$, confirm this finding. Specifically, in the pre-resurfacing case there is a negative correlation between the number of hits and riding speed; this is moderate for low-severity hits, and rather weak for higherseverity ones. So in the before-case, when riding slowly each defect will be hit and felt, but when riding faster the impact of some of the smaller defects may be felt, not as multiple low severity hits, but rather as a single (or a smaller number of) moderate or even high severity hit (e.g. three low-severity hits may be felt as a single moderate one). This does not apply to the more severe defects, though, which will be felt in the same way (moderate or high) regardless of the speed.

After resurfacing, however, a strong positive correlation can be identified between the number of hits and the speed for all three severity levels. This pattern is more in line with what would be anticipated from a "typical" road (with few defects), i.e. that slower cyclists (typically less experienced and on leisure trips, e.g. children) experience a more comfortable ride than faster ones (typically more proficient, e.g. commuters). It also highlights the overall finding of this analysis, which is that in the absence of widespread pavement defects, riding comfort is higher, with slower cyclists benefitting most. On the other hand, the presence of widespread pavement defects/damage results in a deterioration of riding comfort for fast and slow cyclists alike.

\section{CONCLUSION}

The present study has analyzed the impact of pavement defects on cycling comfort using data collected by the iBike, instrumented bicycle, from Southampton's MRE site. From the raw data, vibration hit counts and magnitude values have been determined, and have been compared with perceived real-time cyclist discomfort occurrences, recorded by means of a push button located on the iBike's handlebar. The results show that the number and magnitude of the vibration hits have considerably reduced post-resurfacing of the MRE site, and that instances of high-severity hits are predominantly limited to locations that have either not been resurfaced or have finishing "snags". Moreover, an analysis of the correlation of the riding speed and the number of hits has shown that widespread pavement defects, as in the case of MRE pre-resurfacing, appear to have a detrimental impact on cycling comfort for both faster and slower cyclists.

But while this study has thrown some light into the generally under-explored topic of cycling comfort, there are limitations and work in this direction continues with a view of addressing them. Specifically, considering the collection of data on the perceived cycling comfort, it is currently not possible to enable participants to distinguish between vibration hits of different severity categories in real-time, so in future work, a mechanism will be used to also classify the perceived discomfort as of low, moderate or high severity. Moreover, the analysis has so far concentrated only on the vertical acceleration, but a further indicator of riding comfort is also the steering angle (i.e. the lateral movement); this will be examined in detail in future work.
Furthermore, the vibration hit severity thresholds are currently based on assumptions as a result of a fine-tuning process for simplicity purposes. It planned, however, to better refine their definitions and to relate them to actual comfort levels, as expressed by real cyclists through behavioral surveys. Finally, while the resurfacing of MRE appears to have resulted in decreased journey times and lower values of the average, maximum and standard deviation of the vibration hit magnitude, the data currently only come from a single cyclist at one specific site, and may, therefore, be subject to biases. It is, hence, planned in further work to recruit more test cyclists and survey different sites in order to collect a more diverse dataset so as to be able to draw more generic conclusions.

\section{ACKNOWLEDGMENT}

The authors would like to thank Southampton City Council for funding this project and Transport for London for supplying the Santander Cycle Hire bicycle used in this study.

\section{REFERENCES}

[1] S. Miah, E. Milonidis, I. Kaparias, D. Stirling and N. Karcanias, "Cyclist $360^{\circ}$ Alert: Validation of an Instrumented Bicycle Trajectory Reconstruction Mechanism Using Satellite and Inertial Navigation Systems," UTSG 49th Annual Conference, Dublin, 2017.

[2] M. Bíl, R. Andrášik and J. Kubeček, "How comfortable are your cycling tracks? A new method for objective bicycle vibration measurement," Transportation Research Part C, vol. 56, p. 415-425, 2015

[3] J. Calvey, J. Shackleton, M. Taylor and R. Llewellyn, "Engineering condition assessment of cycling infrastructure: Cyclists' perceptions of satisfaction and comfort," Transportation Research Part A, vol. 78, p. 134-143, 2015.

[4] M. Olieman, R. Marin-Perianu and M. Marin-Perianu, "Measurement of dynamic comfort in cycling using wireless acceleration sensors," Procedia Engineering, vol. 34, p. 568 - 573, 2012.

[5] K. Zang, J. Shen, H. Huang, M. Wan and J. Shi, "Assessing and Mapping of Road Surface Roughness based on GPS and Accelerometer Sensors on Bicycle-Mounted Smartphones," Sensors, vol. 18 , no. $3,2018$.

[6] J. Gao, A. Sha, Y. Huang, L. Hu, Z. Tong and W. Jiang, "Evaluating the cycling comfort on urban roads based on cyclists' perception of vibration," Journal of Cleaner Production, vol. 192, pp. 531-541, 2018.

[7] F. Ayachi, J. Dorey and C. Guastavino, "Identifying factors of bicycle comfort: An online survey with enthusiast cyclists," Applied Ergonomics, vol. 46, pp. 124-136, 2015.

[8] S. Miah, E. Milonidis, I. Kaparias and N. Karcanias, "A novel fusion algorithm to improve localisation accuracy of an instrumented bicycle," in 97th Annual Meeting of the Transportation Research Board, Washington, D.C., 2018.

[9] Southampton City Council, "Cycling Southampton: A strategy for our city 2017-2027," 2016.

[10] Southampton City Council, "My Journey," 2019. [Online]. Available: https://myjourneysouthampton.com. 